\title{
PERFORMANCE EVALUATION OF ROBUST MATCHING MEASURES
}

\author{
Federico Tombari, Luigi Di Stefano, Stefano Mattoccia and Angelo Galanti \\ University of Bologna - Department of Electronics Computer Science and Systems (DEIS) \\ Viale Risorgimento 2, 40136 - Bologna, Italy \\ University of Bologna - Advanced Research Center on Electronic Systems (ARCES) \\ Via Toffano 2/2, 40135 - Bologna, Italy \\ \{federico.tombari,luigi.distefano,stefano.mattoccia\}@unibo.it, angelo.galanti@studio.unibo.it
}

\begin{abstract}
Keywords: $\quad$ Robust matching measure, template matching, performance evaluation.
Abstract: $\quad$ This paper is aimed at evaluating the performances of different measures which have been proposed in literature for robust matching. In particular, classical matching metrics typically employed for this task are considered together with specific approaches aiming at achieving robustness. The main aspects assessed by the proposed evaluation are robustness with respect to photometric distortions, noise and occluded patterns. Specific datasets have been used for testing, which provide a very challenging framework for what concerns the considered disturbance factors and can also serve as testbed for evaluation of future robust visual correspondence measures.
\end{abstract}

\section{INTRODUCTION}

One of the most important tasks in computer vision is visual correspondence, which given two sets of pixels (i.e. two images) aims at finding corresponding pixel pairs belonging to the two sets (homologous pixels). As a matter of fact, visual correspondence is commonly employed in fields such as pattern matching, stereo correspondence, change detection, image registration, motion estimation, image vector quantization.

The visual correspondence task can be extremely challenging in presence of disturbance factors which can typically affect images. A common source of disturbances can be related to photometric distortions between the images under comparison. These can be ascribed to the camera sensors employed in the image acquisition process (due to dynamic variations of camera parameters such as auto-exposure and autogain, or to the use of different cameras), or can be induced by factor extrinsic to the camera, such as changes of the amount of light emitted by the sources or viewing of non-lambertian surfaces at different angles. Other major disturbance factors are represented by distortions of the pixel intensities due to high noise, as well as by the presence of partial occlusions.

In order to increase the reliability of visual cor- respondence many matching measures aimed at being robust with respect to the above mentioned disturbance factors have been proposed in literature. Evaluation assessments have also been proposed which compared some of these measures in particular fields such as stereo correspondence (Chambon and Crouzil, 2003), image registration (Zitová and Flusser, 2003) and image motion (Giachetti, 2000). Generally speaking, apart from classical approaches which will be discussed in Section 2, many robust measures for visual correspondence have been proposed in literature (Tombari et al., 2007), (Crouzil et al., 1996), (Scharstein, 1994), (Seitz, 1989), (Aschwanden and Guggenbuhl, 1992), (Martin and Crowley, 1995), (Fitch et al., 2002), (Zabih and Woodfill, 1994), (Bhat and Nayar, 1998), (Kaneko et al., 2003), (Ullah et al., 2001). A taxonomy which includes the majority of these approaches is proposed in (Chambon and Crouzil, 2003).

This paper focuses on pattern matching, which aims at finding the most similar instances of a given pattern, $P$, within an image.In particular, this paper aims at comparing traditional general purpose approaches together with proposals specifically conceived to achieve robustness, in order to determine which metric is more suitable to deal with disturbance factors such as photometric distortions, noise and occlusions. More precisely, in the comparison 
we will consider the following matching measures: MF (Matching Function), i.e. the approach recently proposed in (Tombari et al., 2007), GC (Gradient Correlation), i.e. the approach initially proposed in (Scharstein, 1994) according to the formulation proposed in (Crouzil et al., 1996) for the pattern matching problem, OC (Orientation Correlation), proposed in (Fitch et al., 2002). Moreover, we will consider the SSD (Sum of Squared Differences) and NCC (Normalized Cross Correlation) measures applied on gradient norms (G-NCC and G-SSD), which showed good robustness against illumination changes in the experimental comparison described in (Martin and Crowley, 1995). The approach relying on gradient orientation only, proposed in (Seitz, 1989) and successively modified in (Aschwanden and Guggenbuhl, 1992), has not been taken into account since, as pointed out in (Crouzil et al., 1996), it is prone to errors in the case of gradient vectors with small magnitude. Considered traditional measures are NCC, ZNCC (Zero-mean NCC) and SSD: NCC and ZNCC showed good robustness with respect to brightness changes, on the other hand SSD showed good insensibility toward noise (Aschwanden and Guggenbuhl, 1992), (Martin and Crowley, 1995).

All the considered proposals are tested on 3 datasets which represent a challenging framework for what regards the considered distortions. These dataset are publicly available ${ }^{1}$ and they might serve as a testbed for future evaluations of robust matching measures. Before reporting on the experimental evaluation, all compared measures are briefly described. Furthermore, for what regards MF, the paper also proposes some modifications to the original approach proposed in (Tombari et al., 2007), and exploits the proposed experimental evaluation to perform a behavioural analysis of this class of functions.

\section{TRADITIONAL MATCHING CRITERIA}

Matching measures traditionally adopted in order to compute the similarity between two pixel sets are typically subdivided into two groups, given they are based on an affinity or distortion criterion. Affinity measures are often based on correlation, with the most popular metric being the Normalized Cross Correlation (NCC). In a pattern matching scenario, being $P$ the pattern vector, sized $M \times N$ (width $\times$ height), $I$ the image vector, sized $W \times H$, and $I_{x y}$ the image subwindow at coordinates $(x, y)$ and having the same dimen-

\footnotetext{
${ }^{1}$ Available at: www.vision.deis.unibo.it/pm-eval.asp
}

sions as $P$, the NCC function at $(x, y)$ is given by:

$$
N C C(x, y)=\frac{\sum_{i=1}^{M} \sum_{j=1}^{N} P(i, j) \cdot I_{x y}(i, j)}{\sqrt{\sum_{i=1}^{M} \sum_{j=1}^{N} P^{2}(i, j)} \cdot \sqrt{\sum_{i=1}^{M} \sum_{j=1}^{N} I_{x y}^{2}(i, j)}}
$$

As it can be seen, the cross-correlation between $P$ and $I_{x y}$ is normalized by the $L_{2}$ norms of the two vectors, in order to render the measure robust to any spatially constant multiplicative bias. By subtracting the mean intensity value of the pattern and of the image subwindow we get an even more robust matching measure:

$$
\operatorname{ZNCC}(x, y)=\frac{\sum_{i=1}^{M} \sum_{j=1}^{N}(P(i, j)-\bar{P}) \cdot\left(I_{x y}(i, j)-\bar{I}_{x y}\right)}{\sqrt{\sum_{i=1}^{M} \sum_{j=1}^{N}(P(i, j)-\bar{P})^{2}} \cdot \sqrt{\sum_{i=1}^{M} \sum_{j=1}^{N}\left(I_{x y}(i, j)-\bar{I}_{x y}\right)^{2}}}
$$

where $\bar{P}$ and $\bar{I}_{x y}$ represent respectively the mean intensity of $P$ and $I_{x y}$. This measure is referred to as Zero-mean NCC (ZNCC) and it is robust to spatially constant affine variations of the image intensities.

As regards the distortion criterion, the classical measures are based on the $L_{p}$ distance between $P$ and $I_{x y}$. In particular, with $p=2$ we get the Sum of Squared Differences (SSD):

$$
\operatorname{SSD}(x, y)=\sum_{i=1}^{M} \sum_{j=1}^{N}\left(P(i, j)-I_{x y}(i, j)\right)^{2}
$$

While all these measures are usually computed directly on the pixel intensities of the image sets, in (Martin and Crowley, 1995) it was shown that by computing these measures on the gradient norm of each pixel a higher robustness is attained, i.e. for what concerns insensitivity to illumination changes G-SSD and G-NCC showed to perform well. In particular, if we denote with $G_{P}(i, j)$ the gradient of the pattern at pixel $(i, j)$ :

$$
G_{P}(i, j)=\left[\frac{\partial P(i, j)}{\partial i}, \frac{\partial P(i, j)}{\partial j}\right]^{T}=\left[G_{i}^{P}(i, j), G_{j}^{P}(i, j)\right]^{T}
$$

and similarly with $G_{I_{x y}}(i, j)$ the gradient of the image subwindow at pixel $(i, j)$ :

$$
G_{I_{x y}}(i, j)=\left[\frac{\partial I_{x y}(i, j)}{\partial i}, \frac{\partial I_{x y}(i, j)}{\partial j}\right]^{T}=\left[G_{i}^{I_{x y}}(i, j), G_{j}^{I_{x y}}(i, j)\right]^{T}
$$

the gradient norm, or magnitude, in each of the two cases is computed as:

$$
\begin{gathered}
\left\|G_{P}(i, j)\right\|=\sqrt{G_{i}^{P}(i, j)^{2}+G_{j}^{P}(i, j)^{2}} \\
\left\|G_{I_{x y}}(i, j)\right\|=\sqrt{G_{i}^{I_{x y}}(i, j)^{2}+G_{j}^{I_{x y}}(i, j)^{2}}
\end{gathered}
$$

i.e. $\|\cdot\|$ represents the $L_{2}$ norm of a vector. Hence the G-NCC function can be defined as:

$$
G-N C C(x, y)=\frac{\sum_{i=1}^{M} \sum_{j=1}^{N}\left\|G_{P}(i, j)\right\| \cdot\left\|G_{I_{x y}}(i, j)\right\|}{\sqrt{\sum_{i=1}^{M} \sum_{j=1}^{N}\left\|G_{P}(i, j)\right\|^{2}} \cdot \sqrt{\sum_{i=1}^{M} \sum_{j=1}^{N}\left\|G_{I_{x y}}(i, j)\right\|^{2}}}
$$


and the G-SSD function as:

$$
G-\operatorname{SSD}(x, y)=\sum_{i=1}^{M} \sum_{j=1}^{N}\left(\left\|G_{P}(i, j)\right\|-\left\|G_{I_{x y}}(i, j)\right\|\right)^{2}
$$

Since (Martin and Crowley, 1995) recommends to compute the partial derivatives for the gradient computation on a suitably smoothed image, in our implementation they will be computed by means of the Sobel masks.

\section{THE MF MEASURE}

In (Tombari et al., 2007) a matching measure was proposed which is implicitly based on the so-called ordering constraint, that is, under the assumption that photometric distortions do not affect the order between intensities of neighbouring pixels. This is analogous to the assumption that the sign of the difference between a pair of neighbouring pixels should not change in presence of this kind of distortions. For this reason the proposed measure correlates the differences between a set of pixel pairs defined on $P$ and their correspondent ones on $I_{x y}$. In particular, the pairs in each set include all pixels at distance 1 and 2 one to another along horizontal and vertical directions. In order to compute this set, we define a vector of pixel differences computed at a point $(i, j)$ on $P$ :

$$
\delta_{\mathbf{1}, \mathbf{2}}^{\mathbf{P}}(i, j)=\left[\begin{array}{c}
P(i-1, j)-P(i, j) \\
P(i, j-1)-P(i, j) \\
P(i-1, j)-P(i+1, j) \\
P(i, j-1)-P(i, j+1)
\end{array}\right]
$$

and, similarly, at a point $(i, j)$ on $I_{x y}$ :

$$
\delta_{\mathbf{1 , 2}}^{\mathbf{I}_{\mathbf{x y}}}(i, j)=\left[\begin{array}{c}
I_{x y}(i-1, j)-I_{x y}(i, j) \\
I_{x y}(i, j-1)-I_{x y}(i, j) \\
I_{x y}(i-1, j)-I_{x y}(i+1, j) \\
I_{x y}(i, j-1)-I_{x y}(i, j+1)
\end{array}\right]
$$

Hence, the MF function proposed in (Tombari et al., 2007) consists in correlating these two vectors for each point of the pattern and the subwindow, and in normalizing the correlation with the $L_{2}$ norm of the vectors themselves:

$$
M F_{1,2}(x, y)=\frac{\sum_{i=1}^{M} \sum_{j=1}^{N} \delta_{\mathbf{1}, \mathbf{2}}^{\mathbf{P}}(i, j) \circ \delta_{\mathbf{1}, \mathbf{2}}^{\mathbf{I}_{\mathbf{y}}}(i, j)}{\sqrt{\sum_{i=1}^{M} \sum_{j=1}^{N} \delta_{\mathbf{1}, \mathbf{2}}^{\mathbf{P}}(i, j) \circ \delta_{\mathbf{1}, \mathbf{2}}^{\mathbf{P}}(i, j)} \cdot \sqrt{\sum_{i=1}^{M} \sum_{j=1}^{N} \delta_{\mathbf{1}, \mathbf{2}}^{\mathbf{I}_{\mathbf{y}}}(i, j) \circ \delta_{\mathbf{1 , 2}}^{\mathbf{I} \mathbf{y}}(i, j)}}
$$

where $\circ$ represents the dot product between two vectors, and the normalization allows the measure to range between $[-1,1]$.

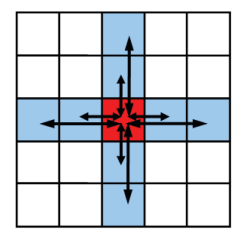

$\mathrm{MF}_{1,2}$

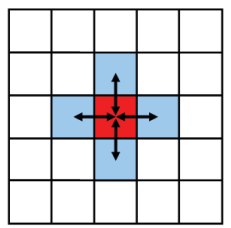

$\mathrm{MF}_{1}$

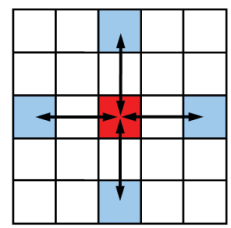

$\mathrm{MF}_{2}$
Figure 1: The 3 considered sets of neighbouring pixel pairs.

It is a peculiarity of this method that, because of the correlation between differences of pixel pairs, intensity edges tend to determine higher correlation coefficients (in magnitude) with respect to low-textured regions. Thus, this can be seen as if the measure mostly relies on the pattern edges. For this reason, MF can be usefully employed also in presence of high levels of noise, as this disturbance factor can typically violate the ordering constraint on low-textured regions, but seldom along intensity edges. Similar considerations can be made in presence of occluded patterns.

The set of pixel pairs originally proposed in (Tombari et al., 2007) can be seen as made out of two subsets: the set of all pixels at distance 1 one to another along horizontal and vertical directions, and the set of all pixels at distance 2 one to another along same directions. Theoretically, the former should benefit of the higher correlation given by adjacent pixels, while the latter should be less influenced by quantization noise that is introduced by the camera sensor. We will refer to the MF measure applied on each of the two subsets as, respectively, $M F_{1}$ and $M F_{2}$. For these last two cases, once defined the pixel differences relative to the case of distance 1 :

$$
\begin{aligned}
\delta_{\mathbf{1}}^{\mathbf{P}}(i, j) & =\left[\begin{array}{c}
P(i-1, j)-P(i, j) \\
P(i, j-1)-P(i, j)
\end{array}\right] \\
\delta_{\mathbf{1}}^{\mathbf{I}_{\mathbf{x y}}}(i, j) & =\left[\begin{array}{c}
I_{x y}(i-1, j)-I_{x y}(i, j) \\
I_{x y}(i, j-1)-I_{x y}(i, j)
\end{array}\right]
\end{aligned}
$$

and the pixel differences relative to the case of distance 2 :

$$
\begin{gathered}
\delta_{\mathbf{2}}^{\mathbf{P}}(i, j)=\left[\begin{array}{c}
P(i-1, j)-P(i+1, j) \\
P(i, j-1)-P(i, j+1)
\end{array}\right] \\
\delta_{\mathbf{2}}^{\mathbf{I}_{\mathbf{x y}}}(i, j)=\left[\begin{array}{c}
I_{x y}(i-1, j)-I_{x y}(i+1, j) \\
I_{x y}(i, j-1)-I_{x y}(i, j+1)
\end{array}\right]
\end{gathered}
$$

it is straightforward to define the two novel measures $M F_{1}$ and $M F_{2}$ as: 


$$
\begin{aligned}
& M F_{1}(x, y)=\frac{\sum_{i=1}^{M} \sum_{j=1}^{N} \delta_{1}^{\mathbf{p}}(i, j) \circ \delta_{1}^{\mathbf{I x y}}(i, j)}{\sqrt{\sum_{i=1}^{M} \sum_{j=1}^{N} \delta_{1}^{\mathbf{P}}(i, j) \circ \delta_{\mathbf{1}}^{\mathbf{P}}(i, j)} \cdot \sqrt{\sum_{i=1}^{M} \sum_{j=1}^{N} \delta_{1}^{\mathbf{I} \mathbf{y}}(i, j) \circ \delta_{1}^{\mathbf{I x y}}(i, j)}} \\
& M F_{2}(x, y)=\frac{\sum_{i=1}^{M} \sum_{j=1}^{N} \delta_{2}^{\mathbf{P}}(i, j) \circ \delta_{2}^{\mathbf{I x y}}(i, j)}{\sqrt{\sum_{i=1}^{M} \sum_{j=1}^{N} \delta_{2}^{\mathbf{P}}(i, j) \circ \delta_{2}^{\mathbf{P}}(i, j)} \cdot \sqrt{\sum_{i=1}^{M} \sum_{j=1}^{N} \delta_{2}^{\mathbf{I x y}}(i, j) \circ \delta_{2}^{\mathbf{I x y}}(i, j)}}
\end{aligned}
$$

A graphical representation of the 3 different pixel pair sets used by $M F_{1,2}, M F_{1}$ and $M F_{2}$ is shown in Fig. 1. We believe that it is interesting to exploit the performance evaluation proposed in this paper also with the aim of determining if there is an optimal set between these 3 or if, conversely, the measure has the same behaviour on all sets. This issue will be discussed in Section 6.

\section{THE OC MEASURE}

The OC measure (Fitch et al., 2002) is based on the correlation of the orientation of the intensity gradient. In particular, for each gradient of the pattern $G_{P}(i, j)$ a complex number representing the orientation of the gradient vector is defined as:

$$
O_{P}(i, j)=\operatorname{sgn}\left(G_{i}^{P}(i, j)+\imath G_{j}^{P}(i, j)\right)
$$

with $\imath$ denoting the imaginary unit and where:

$$
\operatorname{sgn}(x)= \begin{cases}0 & i f|x|=0 \\ \frac{x}{|x|} & \text { otherwise }\end{cases}
$$

Analogously, a complex number representing the orientation of the image subwindow gradient vector $G_{I_{x y}}(i, j)$ is defined as:

$$
O_{I x y}(i, j)=\operatorname{sgn}\left(G_{i}^{I x y}(i, j)+\imath G_{j}^{I x y}(i, j)\right)
$$

As proposed in (Fitch et al., 2002), the partial derivatives for the gradient computation are calculated by approximating them with the central differences. Hence, the OC measure between $P$ and $I_{x y}$ is defined as the real part of the correlation between all gradient orientations belonging to $P$ and $I_{x y}$ :

$$
O C(x, y)=\operatorname{Re}\left\{\sum_{i=1}^{M} \sum_{j=1}^{N} O_{P}(i, j) \cdot O_{I_{x y}}^{*}(i, j)\right\}
$$

with $*$ indicating the conjugate of the complex vector. (Fitch et al., 2002) proposes to compute the correlation operation in the frequency domain by means of the FFT by exploiting the correlation theorem in order to achieve computational efficiency.

\section{THE GC MEASURE}

The GC measure, proposed in (Crouzil et al., 1996) and derived from a measure introduced in (Scharstein, 1994), is based on two terms, referred to as distinctiveness (D) and confidence (C), both computed from intensity gradients:

$$
\begin{array}{r}
D(x, y)=\sum_{i=1}^{M} \sum_{j=1}^{N}\left\|G_{P}(i, j)-G_{I x y}(i, j)\right\| \\
C(x, y)=\sum_{i=1}^{M} \sum_{j=1}^{N}\left(\left\|G_{P}(i, j)\right\|+\left\|G_{I_{x y}}(i, j)\right\|\right)
\end{array}
$$

The GC measure is then defined as:

$$
G C(x, y)=\frac{D(x, y)}{C(x, y)}
$$

Its minimum value is 0 , indicating the pattern is identical to the image subwindow. For any other positive value, the greater the value, the higher the dissimilarity between the two vectors. In order to compute the partial derivatives, (Crouzil et al., 1996) proposes to use either the Sobel operator or the ShenCastan ISEF filter (Shen and Castan, 1992). For ease of comparison with other measures (i.e. G-SSD and G-NCC) in our implementation we will use the formulation based on the Sobel operator.

\section{EXPERIMENTAL COMPARISON}

This section presents the experimental evaluation aimed at assessing the performances of the various measures presented in this paper. The 3 datasets, available at: www.vision.deis.unibo.it/pm-eval.asp, used for our experiments are characterized by a significant presence of the disturbance factors discussed previously, and are now briefly described.

Guitar. In this dataset, 7 patterns were extracted from a picture which was taken with a good camera sensor (3 MegaPixels) and under good illumination conditions given by a lamp and some weak natural light. All these patterns have to be sought in $10 \mathrm{im}$ ages which were taken with a cheaper and more noisy sensor (1.3 MegaPixels, mobile phone camera). Illumination changes were introduced in the images by means of variations of the rheostat of the lamp illuminating the scene $(G 1-G 4)$, by using a torch light instead of the lamp (G5-G6), by using the camera flash instead of the lamp $(G 7-G 8)$, by using the camera flash together with the lamp (G9), by switching off the lamp (G10). Furthermore, additional distortions were introduced by slightly changing the camera position at each pose and by the JPEG compression. 
Table 1: Matching errors reported by the considered measures.

\begin{tabular}{|c||c|c|c||c|}
\hline & Guitar & $M P-I C$ & $M P$-Occl & Total \\
\hline $\mathrm{SSD}$ & $39 / 70$ & $8 / 12$ & $8 / 8$ & $55 / 90$ \\
\hline $\mathrm{G}-\mathrm{SSD}$ & $17 / 70$ & $4 / 12$ & $4 / 8$ & $25 / 90$ \\
\hline $\mathrm{NCC}$ & $27 / 70$ & $5 / 12$ & $8 / 8$ & $40 / 90$ \\
\hline $\mathrm{G}-\mathrm{NCC}$ & $13 / 70$ & $1 / 12$ & $7 / 8$ & $21 / 90$ \\
\hline $\mathrm{ZNCC}$ & $9 / 70$ & $0 / 12$ & $6 / 8$ & $15 / 90$ \\
\hline$M F_{1}$ & $8 / 70$ & $0 / 12$ & $2 / 8$ & $10 / 90$ \\
\hline$M F_{1,2}$ & $5 / 70$ & $0 / 12$ & $1 / 8$ & $6 / 90$ \\
\hline$M F_{2}$ & $5 / 70$ & $0 / 12$ & $1 / 8$ & $6 / 90$ \\
\hline $\mathrm{OC}$ & $13 / 70$ & $1 / 12$ & $1 / 8$ & $15 / 90$ \\
\hline $\mathrm{GC}$ & $6 / 70$ & $2 / 12$ & $0 / 8$ & $8 / 90$ \\
\hline
\end{tabular}

Mere Poulard - Illumination Changes. In dataset Mere Poulard - Illumination Changes (MP-IC), the picture on which the pattern was extracted was taken under good illumination conditions given by neon lights by means of a 1.3 MegaPixels mobile phone camera sensor. This pattern is then searched within 12 images which were taken either with the same camera (prefixed by $G C$ ) or with a cheaper, 0.3 VGA camera sensor (prefixed by $B C$ ). Distortions are due to slight changes in the camera point of view and by different illumination conditions such as: neon lights switched off and use of a very high exposure time $(B C-N 1$, $B C-N 2, G C-N)$, neon lights switched off $(B C-N L$, $G C-N L$ ), presence of structured light given by a lamp light partially occluded by various obstacles $(B C-S T 1$, $\cdots, B C-S T 5)$, neon lights switched off and use of the camera flash $(G C-F L)$, neon lights switched off, use of the camera flash and of a very long exposure time $(G C-N F L)$. Also in this case, images are JPEG compressed.

Mere Poulard - Occlusions. In the dataset Mere Poulard - Occlusions (MP-Occl) the pattern is the same as in dataset MP-IC, which now has to be found in 8 images taken with a 0.3 VGA camera sensor. In this case, partial occlusion of the pattern is the most evident disturbance factor. Occlusions are generated by a person standing in front of the camera $(O P 1, \cdots$, $O P 4$ ), and by a book which increasingly covers part of the pattern $(O B 1, \ldots, O B 4)$. Distortions due to illumination changes, camera pose variations, JPEG compression are also present.

The number of pattern matching instances is thus 70 for the Guitar dataset, 12 for the MP-IC dataset and 8 for the MP-Occl dataset, for a total of 90 instances overall. The result of a pattern matching process is considered erroneous when the coordinates of the best matching subwindow found by a certain measure are further than \pm 5 pixel from the correct ones.

Tab. 1 reports the matching errors yielded by the considered metrics on the 3 datasets. As it can be seen, approaches specifically conceived to achieve robustness generally outperform classical measures, apart from the ZNCC which performs badly in presence of occlusions but shows good robustness in handling strong photometric distortions. The two measures which yield the best performance are MF and $\mathrm{GC}$, with a number of total errors respectively equal to 6 and 8 . In particular, MF performs better on datasets characterized by strong photometric distortions, conversely GC seems to perform better in presence of occlusions.

For what regards the $3 \mathrm{MF}$ measures themselves, it seems clear that the use of differences relative to adjacent pixels suffers of the quantization noise introduced by the camera sensor, hence they are less reliable than differences computed on a distance equal to 2. Moreover, as a consequence of the fact that $M F_{1,2}$ and $M F_{2}$ yield the same results on all datasets, $M F_{2}$ seems the more appropriate MF measure since it requires only 2 correlation terms instead of the 4 needed by $M F_{1,2}$. Finally, for what regards traditional approaches, it is interesting to note that the application of NCC and SSD on the gradient norms rather than on the pixel intensities allows for a significantly higher robustness throughout all the considered datasets. 


\section{CONCLUSIONS AND FUTURE WORKS}

An experimental evaluation of robust matching measure for pattern matching has been presented. We have compared traditional approaches with proposals specifically aimed at achieving robustness in presence of disturbance factors such as photometric distortions, noise, occlusions. The evaluation conducted on a challenging dataset has pointed out that the best performing metric is MF followed by GC, which were able to achieve an error rate of respectively $6.7 \%$ and $8.9 \%$. The experiments have also shown that a modified version of the MF measure consisting of only 2 correlation terms instead of 4 allows for achieving equivalent results with respect to the original formulation introduced in (Tombari et al., 2007).

Future works aims at extending the proposed comparison to other measures not specifically proposed for the pattern matching problem, and also at enriching the dataset used for the evaluation with more images.

\section{REFERENCES}

Aschwanden, P. and Guggenbuhl, W. (1992). Experimental results from a comparative study on correlation-type registration algorithms. In Forstner, W. and Ruwiedel, S., editors, Robust computer vision, pages 268-289. Wichmann.

Bhat, D. and Nayar, S. (1998). Ordinal measures for image correspondence. IEEE Trans. Pattern Recognition and Machine Intelligence, 20(4):415-423.

Chambon, S. and Crouzil, A. (2003). Dense matching using correlation: new measures that are robust near occlusions. In Proc. British Machine Vision Conference (BMVC 2003), volume 1, pages 143-152.

Crouzil, A., Massip-Pailhes, L., and Castan, S. (1996). A new correlation criterion based on gradient fields similarity. In Proc. Int. Conf. Pattern Recognition (ICPR), pages 632-636.

Fitch, A. J., Kadyrov, A., Christmas, W. J., and J, K. (2002). Orientation correlation. In Rosin, P. and Marshall, D., editors, British Machine Vision Conference, volume 1, pages 133-142.

Giachetti, S. (2000). Matching techniques to compute image motion. Image and Vision Computing, 18:247260.

Kaneko, S., Satoh, Y., and Igarashi, S. (2003). Using selective correlation coefficient for robust image registration. Journ. Pattern Recognition, 36(5):1165-1173.

Martin, J. and Crowley, J. (1995). Experimental comparison of correlation techniques. In Proc. Int. Conf. on Intelligent Autonomous Systems, volume 4, pages 8693.
Scharstein, D. (1994). Matching images by comparing their gradient fields. In Proc. Int. Conf. Pattern Recognition (ICPR), volume 1, pages 572-575.

Seitz, P. (1989). Using local orientational information as image primitive for robust object recognition. In Proc. SPIE, Visual Communication and Image Processing $I V$, volume 1199, pages 1630-1639.

Shen, J. and Castan, S. (1992). An optimal linear operator for step edge detection. Graphical Models and Image Processing (CVGIP), 54(2):112-133.

Tombari, F., Di Stefano, L., and Mattoccia, S. (2007). A robust measure for visual correspondence. In Proc. 14th Int. Conf. on Image Analysis and Processing (ICIAP 2007), pages 376-381.

Ullah, F., Kaneko, S., and Igarashi, S. (2001). Orientation code matching for robust object search. IEICE Trans. Information and Systems, E-84-D(8):999-1006.

Zabih, R. and Woodfill, J. (1994). Non-parametric local transforms for computing visual correspondence. In Proc. European Conf. Computer Vision, pages 151158

Zitová, B. and Flusser, J. (2003). Image registration methods: a survey. Image and Vision Computing, 21(11):977-1000. 